\title{
Social Adjustment in Survivors of Acute Lymphoblastic Leukemia without Cranial Radiation Therapy
}

\author{
Fiona Schulte ${ }^{1}$, Erin Merz ${ }^{2}$, K Russell ${ }^{3}$, Courtney Tromburg ${ }^{4}$, Sara Cho ${ }^{1}$, Andrew $\operatorname{Tran}^{3}$, \\ Kathleen Reynolds ${ }^{5}$, and Lianne Tomfohr ${ }^{3}$ \\ ${ }^{1}$ University of Calgary Cumming School of Medicine \\ ${ }^{2}$ California State University Dominguez Hills \\ ${ }^{3}$ University of Calgary \\ ${ }^{4}$ University of Alberta Faculty of Medicine \& Dentistry \\ ${ }^{5}$ Alberta Children's Hospital
}

June 26, 2021

\begin{abstract}
Objective: To evaluate group differences in social adjustment in survivors of pediatric ALL compared to survivor siblings, and controls; identify disease-related predictors of social adjustment in survivors; and explore whether executive functioning explained differences in social adjustment across groups and between disease-related predictors. Methods: Survivors of pediatric $\operatorname{ALL}(\mathrm{n}=38$, average age at diagnosis $=4.27$ years $[\mathrm{SD}=1.97$; average time off treatment $=4.83$ years $[\mathrm{SD}=1.52])$, one sibling (if available, $\mathrm{n}=20$ ), and one parent from each family were recruited from a long-term survivor clinic. Healthy age- and sex-matched controls $(n=38)$ and one parent from each family were recruited from the community. Parents completed the Behavioral Assessment System for Children, Parent Rating Scale (BASC-3) Social Withdrawal subscale as a measure of social adjustment and the Behavior Rating Inventory of Executive Functions (BRIEF-2) as a measure of executive function for each of their children. Results: Parents reported that survivors had significantly worse social adjustment compared to controls $(\mathrm{b}=6.34, \mathrm{p}=.004)$, but not survivor siblings. Among survivors, greater time off treatment $(\mathrm{b}=2.06, \mathrm{p}=.058)$ and poorer executive functioning $(\mathrm{b}=0.42, \mathrm{p}=.006)$ were associated with worse social adjustment. Executive function did not mediate differences in social withdrawal between survivors and controls or the relationship between time off treatment and social withdrawal among survivors. Conclusions: Survivors of pediatric ALL presenting to follow-up programs should be screened for difficulties with social adjustment. Future research should examine treatment- and non-treatment-related factors contributing to poorer social outcomes.
\end{abstract}

RUNNING HEAD: Social Adjustment in Survivors of Pediatric ALL

Social Adjustment in Survivors of Acute Lymphoblastic Leukemia without Cranial Radiation Therapy

Fiona Schulte, PhD,${ }^{1,2}$ Erin L. Merz, PhD, MPH, ${ }^{3}$ K. Brooke Russell, MSc, ${ }^{4}$ Courtney Tromburg, $\mathrm{BA}^{5}$ Sara Cho, BSc ${ }^{1}$ Andrew Tran, ${ }^{4}$ Kathy Reynolds, MD, ${ }^{2,6}$ Lianne Tomfohr-Madsen, $\mathrm{PhD}^{4}$

${ }^{1}$ Department of Oncology, Division of Psychosocial Oncology, Cumming School of Medicine, University of Calgary, Calgary, AB

${ }^{2}$ Alberta Children's Hospital, Hematology, Oncology, and Transplant Program, Calgary, AB

${ }^{3}$ Department of Psychology, California State University, Dominguez Hills, Carson, CA, USA

${ }^{4}$ Department of Psychology, University of Calgary, Calgary, AB 
${ }^{5}$ Faculty of Medicine, University of Alberta

${ }^{6}$ Department of Family Medicine, University of Calgary, Calgary, AB

Corresponding Author: Fiona Schulte, Ph.D. Assistant Professor, Department of Oncology, Division of Psychosocial Oncology, Cumming School of Medicine, University of Calgary

22022 St SW, Calgary, AB T2S 3C3; Tel: 403-698-8103; Email: Fiona.schulte@ahs.ca

\begin{tabular}{ll}
\hline Abbreviations & Definition \\
\hline ALL & Acute Lymphoblastic Leukemia \\
SD & Standard Deviation \\
CNS & Central Nervous System \\
CRT & Cranial Radiation Therapy \\
TBI & Traumatic Brain Injury \\
BASC-3 & Behavioral Assessment System for Children, Third Edition \\
BRIEF-2 & Behavior Rating Inventory of Executive Functions \\
\hline
\end{tabular}

\section{Abstract}

Objective: To evaluate group differences in social adjustment in survivors of pediatric ALL compared to survivor siblings, and controls; identify disease-related predictors of social adjustment in survivors; and explore whether executive functioning explained differences in social adjustment across groups and between disease-related predictors. Methods: Survivors of pediatric ALL $(n=38$, average age at diagnosis=4.27 years $[S D=1.97]$; average time off treatment $=4.83$ years $[S D=1.52]$ ), one sibling (if available, $\mathrm{n}=20$ ), and one parent from each family were recruited from a long-term survivor clinic. Healthy age- and sex-matched controls $(n=38)$ and one parent from each family were recruited from the community. Parents completed the Behavioral Assessment System for Children, Parent Rating Scale (BASC-3) Social Withdrawal subscale as a measure of social adjustment and the Behavior Rating Inventory of Executive Functions (BRIEF-2) as a measure of executive function for each of their children. Results: Parents reported that survivors had significantly worse social adjustment compared to controls $(b=6.34, p=.004)$, but not survivor siblings. Among survivors, greater time off treatment $(b=2.06, p=.058)$ and poorer executive functioning $(b=0.42$, $p=.006)$ were associated with worse social adjustment. Executive function did not mediate differences in social withdrawal between survivors and controls or the relationship between time off treatment and social withdrawal among survivors. Conclusions: Survivors of pediatric ALL presenting to follow-up programs should be screened for difficulties with social adjustment. Future research should examine treatment- and non-treatment-related factors contributing to poorer social outcomes.

Keywords: ALL, Late effects, Pediatric oncology, Psychology, Psychosocial

Social Adjustment in Survivors of Pediatric ALL

Pediatric ALL is a major medical success story. Advances in treatment for ALL have resulted in one of the most impressive increases in cancer survival over the last 35 years, from $60 \%$ in 1975 to approximately 80 90\% today in North America. ${ }^{1}$ The number of survivors is growing rapidly. Given the dramatically improved survival rates, efforts were made in the early 1990's to reduce the toxicity of treatment to the central nervous system (CNS) in ALL. Modern treatments for pediatric ALL rely less on cranial radiation therapy (CRT) for CNS prophylaxis and instead focus on intermediate or high dose intravenous methotrexate and intrathecal chemotherapies, intended to maximize CNS drug exposure while reducing systemic drug toxicity. ${ }^{2} \mathrm{ALL}$ is one of few pediatric cancers to receive both intravenous methotrexate and intrathecal chemotherapy. Despite efforts to reduce CNS toxicity, negative effects of these therapies on the developing brain persist. ${ }^{3-5}$

Survivors of pediatric ALL experience difficulties with social adjustment. ${ }^{6-8}$ Social adjustment is defined as the extent to which children attain socially desirable and developmentally appropriate goals. ${ }^{9}$ The social 
adjustment difficulties that have been reported in the literature for survivors of pediatric ALL include being more withdrawn, socially isolated, lonely, and bullied compared to their siblings and classmates ${ }^{8,10}$ Survivors of ALL have been found to engage in less than half the number of social activities than their peers. ${ }^{8,10-12}$ As adults, survivors of ALL are much less likely to marry or live independently compared to their siblings. ${ }^{6,13-16}$ Tied to these enduring social difficulties, adult survivors of pediatric ALL are 1.7 times more likely to report mental health problems compared to their siblings, placing a significant burden on our health, economic, and social welfare systems. ${ }^{15}$ The majority of studies describing these specific social outcomes, however, involved patients treated with older protocols from the late 1970s through the mid-1980s that included CRT. Moreover, many of these studies reported the outcomes of long-term survivors of ALL. We do not yet know what effect modern therapies, which include higher doses of intravenous methotrexate and intrathecal chemotherapies, have on social adjustment, particularly in the early survivorship period (e.g., 2-7 years post treatment).

CNS-directed chemotherapies impair cognitive-executive processes. ${ }^{17-21}$ Despite the effort to reduce toxicity to the developing brain by eliminating CRT, treatment with methotrexate and intrathecal chemotherapy alone has been linked to the presence of cognitive deficits in 40-60\% of survivors of ALL. ${ }^{3,19-21}$ Cognitive deficits experienced by survivors include difficulties with tasks related to processing speed, attention, working memory, and executive function. Consistent with the Social Competence Model developed by Yeates and colleagues, ${ }^{9}$ these same cognitive processes may play a role in social adjustment. The 'Social Competence Model' is a theoretical framework examining correlates of social adjustment in children with acquired brain injuries. ${ }^{9}$ This model takes a multilevel approach to understanding social competence, distinguishing among three key levels: 1) social adjustment; 2) social interaction; and 3) social information processing, comprised of social-cognitive and cognitive-executive function and assumes significant associations among these levels. Indeed, attention has been found to be uniquely associated with social adjustment in survivors of pediatric ALL relative to other known demographic- and treatment-related risk factors. ${ }^{22}$ The social competence model has been validated in research examining social outcomes among a variety of pediatric brain disorders, including traumatic brain injury (TBI), ${ }^{23,24}$ stroke,${ }^{25}$ epilepsy,${ }^{26}$ and survivors of pediatric brain tumor. ${ }^{27}$ The contribution of cognitive-executive processes to social adjustment in survivors of ALL requires further investigation.

Thus, the specific aims of this study were to evaluate: 1) potential group differences in social adjustment across three groups of children (survivors of ALL, survivor siblings, age- and sex-matched controls); 2) disease-related factors (age at diagnosis, time off treatment) and executive functioning as predictors of social adjustment in survivors; 3 ) whether executive functioning mediates the differences in social adjustment across groups that were identified in the first aim (i.e., executive functioning as a mediator of the relationship between group and social adjustment); and 4) whether executive functioning mediates the relationship between the disease-related predictors (that were identified in the second aim) and social adjustment in survivors (i.e., executive functioning as a mediator of the relationship between disease-related predictors and social adjustment). We hypothesized that survivors of ALL would demonstrate significantly more social adjustment difficulties compared to their siblings and controls and that disease-related predictors of social adjustment would include diagnostic risk status with survivors diagnosed with high-risk disease being more likely to report social adjustment difficulties. Finally, we hypothesized that executive functioning would explain the differences in social adjustment across groups and disease-related predictors.

\section{Methods}

The methods described in this study are part of a larger study. ${ }^{28}$ For the purposes of this manuscript, we only included measures and procedures relevant to the current study.

ParticipantsEthics approval for this study was obtained through the local institutional review board (HREBA.CC-16-0268). Survivors of pediatric ALL, one sibling (if available, between 8-18 years of age), and one parent from each family were recruited through the Long-Term Survivor Clinic at the Alberta Children's Hospital. Eligible families were approached during regular clinic visits by a member of the research team to introduce the study and evaluate their interest in participating. Eligibility criteria for survivors 
included: 1) diagnosed with pediatric ALL and received treatment; 2) 8-18 years of age at the time of the study; 3) were between 2-7 years post-treatment. Exclusion criteria included: 1) diagnosed with a developmental disorder; 2) unable to speak and read English; or 3) treated with CRT. If a survivor did not have an eligible sibling, they were not excluded from participation. Healthy controls, one sibling (if available), and one parent from each family were recruited through the community via online and community advertisements, public and private school mailing lists, and local community hubs and events. The only inclusion criteria for healthy controls and their siblings was that participants were 8-18 years of age at the time of the study. Exclusion criteria included: 1) diagnosed with a chronic illness; 2) diagnosed with a developmental disorder; 3) unable to speak and read English. Healthy controls were sex and age matched to a participant from the survivor group post-hoc.

ProcedureEligible survivors and their parent(s) were approached by a member of the study team at their regular long-term follow-up appointments. Interested families provided written consent and assent. Parents were emailed unique URL links to complete the questionnaire package online via Qualtrics and were asked to complete the package within 7 days. Relevant clinical information was collected from the medical records of all survivors enrolled in the study. Interested potential control participants contacted the study team through the email or telephone number provided on recruitment materials and were screened for eligibility. All eligible control participants completed a one-time visit at the University of Calgary. During this visit, written consent and assent was obtained, and parents completed the questionnaire package on a computer. All participants were renumerated $\$ 15$ after completing questionnaires.

\section{Measures}

Social Adjustment. Social adjustment was measured using the Withdrawal subscale of the Behavioral Assessment System for Children, Third Edition, Parent Rating Scales (BASC-3). ${ }^{29}$ The BASC-3 is a standardized parent-report measure of behavioral and emotional functioning in children and adolescents. The Withdrawal subscale is comprised of 10 items scored on a 5 -point scale $(1=$ never, $5=$ almost always $)$ which are summed to provide a total subscale score. Higher scores reflect higher social withdrawal. The BASC-3 has evidence of validity for use with pediatric cancer survivors and is a reliable measure to detect differences between survivors and healthy controls. ${ }^{30}$ BASC- 3 scores are standardised for age. During statistical analyses, we used the standard t-scores of participants for analyses which have a mean of 50 and a standard deviation of 10. In addition, we considered the frequency of participants who scored above the clinical cut-off $(>60)$ on the BASC-3 Social Withdrawal scale as a measure of those experiencing clinically significant social difficulties. ${ }^{29}$ Alpha was .87 for the child scale (participants age 8-11) and .84 for the adolescent scale (participants age 12-18) in the current sample.Executive Functioning. Executive functioning was measured using the total score of the Behavior Rating Inventory of Executive Functions, Second Edition (BRIEF-2) Parent Form. ${ }^{31}$ The BRIEF-2 is a 59-item parent-report measure of executive function. Items are scored on a 5 -point scale $(1=$ never, $5=$ almost always $)$ which are summed to provide a total score. Higher scores indicate greater deficits in executive function. BRIEF-2 scores are standardised for age. During statistical analyses, we used the standard $t$ - scores of participants for analyses which have a mean of 50 and a standard deviation of 10. Alpha was .97 in the current sample.Demographic information. Participating parents completed a demographic questionnaire which collected the following information: child(s) sex, age, and ethnicity, annual household income, and highest level of parental and maternal education. Clinical information was collected for all survivors of ALL from their medical records including: age at diagnosis, time since diagnosis, time off treatment, and diagnostic risk level (low, standard, intermediate).Statistical AnalysesThe sample for this analysis $(N=96)$ was comprised of 38 survivors, 20 survivor siblings, and 38 age- and sex-matched controls. Data were analyzed using SPSS 26 using a familywise alpha rate of .05. Descriptive statistics were calculated for the full sample.

For aim 1, multilevel modeling was used to evaluate group differences (survivor, survivor sibling, control) in BASC-3 Social Withdrawal. The repeated measures formulation within SPSS Mixed Models was used to handle potential systematic variation (i.e., dependence) within survivor families. ${ }^{32}$ This dependence was considered 'nuisance' variability to be disentangled from the fixed parameters (i.e., mean BASC-3 differences 
between groups), which were of substantive interest. This method models nonindependence at the level of the family as a covariance by allowing for error terms from each child within the same family to be correlated. For aim 2, a main effects regression model was tested with age at diagnosis, time off treatment, and BRIEF-2 score as predictors of BASC-3 Social Withdrawal in survivors only. For aim 3, a mediation model was tested with the significant group pairwise comparisons from aim 1 as the antecedent variable, BRIEF-2 score as the mediator, and BASC-3 Social Withdrawal as the outcome. See Fig. 1 for a description of the tested model. For aim 4 a mediation model was tested with the significant disease-related predictor(s) from aim 2 as the antecedent variable(s), BRIEF-2 score as the mediator, and BASC-3 Social Withdrawal as the outcome in survivors only. The PROCESS macro ${ }^{33}$ for SPSS was used to test the mediation models; the statistical significance of the indirect effect was tested using 5,000 bootstrapped samples.

\section{Results}

Sample characteristics are summarized Table 1. BRIEF-2 scores suggested that children in each group generally had adequate executive functioning. BASC-3 Social Withdrawal scores also suggested that children in each group generally had adequate social adjustment. Approximately one quarter of survivors and survivor siblings and ten percent of controls were considered at risk for poor social adjustment. Among survivors, approximately 7.5 years had passed since their diagnosis, with the average age of diagnosis being around age 4. Most survivors had been off treatment for almost 5 years, and the majority had a standard diagnostic risk level.

\section{Aim 1: Does social adjustment differ across group?}

There was a significant fixed effect for group, $\mathrm{F}(2,78.93)=4.59 p=.013)$. Evaluation of the between-group differences suggested that survivors and their siblings did not significantly differ on BASC-Social Withdrawal scores, $b=2.24(S E=2.39), t(48.89)=0.94, p=.353$. Survivors did have significantly higher BASC-3 Social Withdrawal scores compared to controls, $b=6.34(S E=2.12), t(76)=3.00, p=.004$. Although differences between siblings and controls did not meet the threshold for statistical significance, $b=4.10(S E=2.39), t$ $(48.89)=1.72, p=.092$, the trend suggested slightly higher scores for siblings. Together, these results suggest that survivors had poorer social adjustment compared to age- and sex-matched controls.

\section{Aim 2: What are the disease-related predictors of social adjustment for survivors of ALL?}

The overall model suggested that a significant amount of overall variance in BASC-3 Social Withdrawal scores was explained by age at diagnosis, time off treatment, and BRIEF-2 score: $F(3,33)=3.54, p=.025$, $R^{2}=.24$. Age at diagnosis was not associated with BASC-3 Social Withdrawal scores $(b=0.20, p=.801)$. Time off treatment approached significance with a medium-sized effect $(b=2.06, p=.058, \beta=.30)$, and BRIEF-2 scores were statistically significant with a medium-to-large effect $(b=0.42, p=.006, \beta=.45)$. In sum, these findings suggest, among survivors, greater time off treatment and higher BRIEF-2 scores (i.e., poorer executive functioning) were associated with higher BASC-3 Social Withdrawal scores (i.e., poorer social adjustment).

Aim 3: Does executive functioning explain differences in social adjustment across survivors of ALL and controls?

The significant pairwise differences for BASC-3 Social Withdrawal (survivors vs. controls) gleaned in the first aim were retained for the third aim.11Because the groups being compared (survivor vs. control) were from separate families, the multilevel approach was not needed and a traditional ordinary least squares approach was used. The theoretical model is depicted in Fig. 1. The direct path representing mean differences in BASC-3 Social Withdrawal scores in survivors vs. controls (path c) was statistically significant $b=-6.09, p$ $=.006$. The path representing mean differences in BRIEF-2 scores in survivors vs. controls (path a) was not significant, $b=-2.50, p=.299$. The path representing the relationship between BRIEF-2 scores and BASC-3 Social Withdrawal scores (path b) was significant, $b=0.29, p=.005$. The path representing mean differences in BASC-3 Social Withdrawal scores in survivors vs. controls when BRIEF-2 scores were included in the model (path c') was significant $b=-5.36, p=.012$. The indirect effect of the survivor vs. control group 
differences in BASC-3 Social Withdrawal scores as mediated by BRIEF-2 scores was not significant, $b=-$ $0.73,95 \%$ CI $(-2.60,0.57)$. This suggests that executive functioning does not explain differences in social adjustment between survivors and controls, but rather that the relationship appears to be direct.

\section{Aim 4: Does executive functioning explain the link between disease-related variables and social adjustment in survivors of ALL?}

The path representing the relationship between the antecedent of time off treatment and the mediator of BRIEF-2 score (path a) was not significant, $b=-1.25, p=.305$. The path representing the relationship between the mediator of BRIEF-2 score and the outcome of BASC-3 Social Withdrawal score (path b) was significant, $b=0.42, p=.006$. The direct path representing between the antecedent of time off treatment and the outcome of BASC-3 Social Withdrawal score (path c) was not significant, $b=1.51, p=.186$. The path representing the relationship between the antecedent of time off treatment and the outcome of BASC-3 Social Withdrawal when BRIEF-2 scores were included in the model (path c') approached significance, $b$ $=2.03, \mathrm{p}=.056$. However, the indirect effect of time off treatment on BASC-3 Social Withdrawal through BRIEF-2 was not significant $b=-.53,95 \%$ CI [-1.60, 0.41]. This suggests that executive functioning does not explain the relationship between time off treatment and social adjustment, but rather the relationship between each predictor and social adjustment appear to be direct.

\section{Discussion}

The aims of the current study were to evaluate group differences in social adjustment across three groups of children (survivors of ALL, survivor siblings, controls, control siblings), to examine disease-related predictors of social adjustment in survivors, and to explore executive functioning as a potential mediator of these relationships. Results suggested that survivors of ALL had greater social withdrawal (i.e., poorer social adjustment) compared to controls and the siblings of those controls. This finding was consistent with our hypothesis and with previous literature that has identified social adjustment difficulties among survivors of pediatric ALL. ${ }^{6-8}$ Importantly, when we examined the frequency of those reporting social difficulties within the clinical range (i.e., >60) $26 \%$ of survivors did report social withdrawal within the clinical range compared to only $10 \%$ of controls. Thus, the social adjustment of survivors of ALL warrants greater clinical and research attention.

Importantly, our hypothesis that executive functioning would be associated with social adjustment among survivors as proposed by the Social Competence Model,${ }^{9}$ was supported by our second aim. The current study provides preliminary evidence for the application of the Social Competence Model to survivors of pediatric ALL. What is unique about the current study from previous literature, however, is that none of our current sample of survivors of ALL had received radiotherapy. This finding suggests, therefore, that despite a move towards less toxic therapy for survivors of ALL, social adjustment difficulties remain.

The story does appear to be slightly more complex, however, among survivors of ALL. Although executive function emerged as a significant predictor of social adjustment among survivors of ALL, executive function did not explain differences in social withdrawal between survivors, siblings and controls. This has important implications for the Social Competence Model as well as for our understanding of social adjustment difficulties in survivors of ALL. First, our findings strengthen the applicability of the Social Competence Model by confirming the relationship of executive function to social adjustment, irrespective of acquired brain injury. In other words, this relationship holds among clinical and healthy populations. In order to understand the difference in social adjustment scores between survivors and healthy controls, therefore, consideration of treatment-related variables may be key. For example, it might be important to consider chemotherapy dosage. In addition, little research has explored the indirect effects of a diagnosis of ALL and the consequences of the several years of treatment required to cure ALL and the implications this might have on social development.

The literature exploring non-treatment factors associated with social adjustment in survivors of pediatric ALL is more variable. Some evidence suggests that demographic variables such as ethnicity, marital status, parent education, and household income may play a role in social adjustment outcomes. ${ }^{34-37}$ The literature examining sex and social adjustment outcomes in survivors of ALL has revealed inconsistent findings. ${ }^{22,37,38}$ 
Individual and family factors, such as survivor and parent mental health (e.g., mood, anxiety, posttraumatic growth), ${ }^{39,40}$ and parenting stress ${ }^{36}$ have been associated with social adjustment in this population and require further investigation. Importantly, given our current findings, it would be critical to also explore how such family factors may be related to sibling social adjustment. Knowledge about both treatment and nontreatment factors associated with social adjustment are important to inform risk-based screening guidelines and targets for intervention.

One of the most surprising findings from the study was that survivors did not differ in their social adjustment compared to their siblings. This finding was not consistent with our hypotheses. Certainly, the siblings of pediatric cancer patients are not immune to the experience of cancer within the family and have been shown to exhibit increased levels of emotional distress and behavioral problems. ${ }^{41,42}$ In addition, previous research among a heterogenous sample of pediatric cancer survivors found no differences between survivors and siblings with respect to social adjustment. ${ }^{43}$ It is important to keep in mind, however, our population of survivors in the acute survivorship phase (2-7 years post treatment). Knowing the extensive literature documenting the delayed onset of cognitive deficits, so too might we expect to see the social adjustment of survivors worsen the further off treatment they might be.

This study was not without limitations. To begin, we had small samples to test for these effects, specifically for the survivor sibling group. This study is limited by its cross-sectional design which precludes any conclusions to be made about causal factors of social adjustment difficulties. Mediation models typically imply a sequence of events occurring over time; in the current study we were not able to capture potential 'true' mediation given this design limitation. Longitudinal research is needed to explore the trajectory of social adjustment difficulties for survivors of pediatric ALL and to being to explore mechanisms that may explain this relationship. In addition, although the control groups were a relative strength of the study, we did not compare survivors of ALL to survivors of cancer who do not receive toxic therapies directed at the CNS. Survivors of childhood cancer who did not receive toxic therapies directed at the CNS would have provided a more conservative control group as they have both experienced a traumatic cancer diagnosis and been treated with chemotherapies that lead to socially limiting side effects (e.g., hair loss and compromised immunity limiting their ability to be in public places or school environments). However, without having received therapies believed to be centrally neurotoxic, the effects of CNS-directed treatment on social adjustment could have been more clearly differentiated in survivors of ALL. In addition, while we were able to examine outcomes of survivors based on disease risk status, we did not have specific dosage information for therapies known to be neuro-toxic, including methotrexate and intrathecal chemotherapies. Finally, the study did not explore non-treatment-related factors that might also contribute to social functioning in this population. Future research should aim to explore the other factors that might be related to social function among this population with a specific focus on both treatment- and non-treatment-related factors.

In conclusion, survivors of pediatric ALL are at risk of more social difficulties compared to non-cancer controls. Predictors of social adjustment among survivors included executive functioning and time off treatment. However, executive functioning did not explain any of the observed group differences in social adjustment. This is an important finding among a sample of survivors 2-7 years from the completion of therapy who did not receive CRT and highlights the potential persistent toxicity of therapy for pediatric ALL despite efforts to reduce treatment-related toxicity. Based on the current findings, survivors of pediatric ALL presenting to follow-up programs should be screened for difficulties with social adjustment. Future research should aim to more comprehensively examine the potential multiple factors that may contribute to poorer social adjustment outcomes in order to identify modifiable targets for interventions.

Acknowledgements: This work was supported by the Alberta Children's Hospital Research Institute 2015-16 ARC Award in Family Centered Care.

Conflict of Interest Statement

None to disclose.

Data Availability Statement 
The data that support the findings of this study are available from the corresponding author upon reasonable request.

References

1. Phillips SM, Padgett LS, Leisenring WM, et al. Survivors of childhood cancer in the United States: prevalence and burden of morbidity. Cancer Epidemiol Biomarkers Prev. 2015;24(4):653-663.

2. Cooper SL, Brown PA. Treatment of pediatric acute lymphoblastic leukemia. Pediatr Clin North Am. 2015;62(1):61-73.

3. van der Plas E, Nieman BJ, Butcher DT, et al. Neurocognitive Late Effects of Chemotherapy in Survivors of Acute Lymphoblastic Leukemia: Focus on Methotrexate. J Can Acad Child Adolesc Psychiatry.2015;24(1):25-32.

4. Kerr JZ, Berg S, Blaney SM. Intrathecal chemotherapy. Crit Rev Oncol Hematol. 2001;37(3):227-236.

5. Phillips NS, Cheung YT, Glass JO, et al. Neuroanatomical abnormalities related to dexamethasone exposure in survivors of childhood acute lymphoblastic leukemia. Pediatr Blood Cancer.2019:e27968.

6. Gurney JG, Krull KR, Kadan-Lottick N, et al. Social Outcomes in the Childhood Cancer Survivor Study Cohort. Journal of Clinical Oncology. 2009;27(14):2390-2395.

7. Gunn ME, Lahteenmaki PM, Puukko-Viertomies LR, Henriksson M, Heikkinen R, Jahnukainen K. Potential gonadotoxicity of treatment in relation to quality of life and mental well-being of male survivors of childhood acute lymphoblastic leukemia. J Cancer Surviv.2013;7(3):404-412.

8. Schultz KAP, Ness KK, Whitton J, et al. Behavioral and social outcomes in adolescent survivors of childhood cancer: a report from the childhood cancer survivor study. Journal of clinical oncology : official journal of the American Society of Clinical Oncology.2007;25:3649-3656.

9. Yeates KO, Bigler ED, Dennis M, et al. Social outcomes in childhood brain disorder: A heuristic integration of social neuroscience and developmental psychology. Psychological Bulletin.2007;133:535-556.

10. Noll RB, Bukowski WM, Davies WH, Koontz K, Kulkarni R. Adjustment in the peer system of adolescents with cancer: A two-year study.Journal of Pediatric Psychology. 1993;18:351-364.

11. Katz LF, Leary A, Breiger D, Friedman D. Pediatric cancer and the quality of children's dyadic peer interactions. J Pediatr Psychol. 2011;36(2):237-247.

12. Pendley JS, Dahlquist LM, Dreyer Z. Body image and psychosocial adjustment in adolescent cancer survivors. J Pediatr Psychol.1997;22(1):29-43.

13. Robison LL. Late effects of acute lymphoblastic leukemia therapy in patients diagnosed at 0-20 years of age. Hematology Am Soc Hematol Educ Program. 2011;2011:238-242.

14. Mitby PA, Robison LL, Whitton JA, et al. Utilization of special education services and educational attainment among long-term survivors of childhood cancer: a report from the Childhood Cancer Survivor Study. Cancer. 2003;97:1115-1126.

15. Mody R, Li S, Dover DC, et al. Twenty-five-year follow-up among survivors of childhood acute lymphoblastic leukemia: a report from the Childhood Cancer Survivor Study. Blood. 2008;111(12):5515-5523.

16. Janson C, Leisenring W, Cox C, et al. Predictors of marriage and divorce in adult survivors of childhood cancers: a report from the Childhood Cancer Survivor Study. Cancer epidemiology, biomarkers $\&$ prevention : a publication of the American Association for Cancer Research, cosponsored by the American Society of Preventive Oncology.2009;18:2626-2635.

17. Schatz J, Kramer JH, Ablin A, Matthay KK. Processing speed, working memory, and IQ: A developmental model of cognitive deficits following cranial radiation therapy. Neuropsychology. 2000;14:189-200. 
18. Mulhern RK, Butler RW. Neurocogntive sequelae of childhood cancers and their treatment. Pediatric Rehabilitation. 2004;7:1-14.

19. Campbell LK, Scaduto M, Sharp W, et al. A meta-analysis of the neurocognitive sequelae of treatment for childhood acute lymphocytic leukemia. Pediatr Blood Cancer. 2007;49(1):65-73.

20. Iyer NS, Balsamo LM, Bracken MB, Kadan-Lottick NS. Chemotherapy-only treatment effects on long-term neurocognitive functioning in childhood ALL survivors: a review and meta-analysis. Blood.2015;126(3):346-353.

21. Krull KR, Cheung YT, Liu W, et al. Chemotherapy Pharmacodynamics and Neuroimaging and Neurocognitive Outcomes in Long-Term Survivors of Childhood Acute Lymphoblastic Leukemia. J Clin Oncol.2016;34(22):2644-2653.

22. Moyer KH, Willard VW, Gross AM, et al. The impact of attention on social functioning in survivors of pediatric acute lymphoblastic leukemia and brain tumors. Pediatric blood \& cancer.2012;59:1290-1295.

23. Robinson KE, Fountain-Zaragoza S, Dennis M, et al. Executive functions and theory of mind as predictors of social adjustment in childhood traumatic brain injury. Journal of neurotrauma.2014;31:18351842 .

24. Heverly-Fitt S, Rubin KH, Dennis M, et al. Investigating a Proposed Model of Social Competence in Children With Traumatic Brain Injuries.J Pediatr Psychol. 2016;41(2):235-243.

25. Anderson V, Gomes A, Greenham M, et al. Social competence following pediatric stroke: contributions of brain insult and family environment.Soc Neurosci. 2014;9(5):471-483.

26. Rantanen K, Eriksson K, Nieminen P. Social competence in children with epilepsy-a review. Epilepsy Behav. 2012;24(3):295-303.

27. Schulte F, Vannatta K, Barrera M. Social problem solving and social performance after a group social skills intervention for childhood brain tumor survivors. Psychooncology. 2014;23(2):183-189.

28. Russell KB, Merz EL, Reynolds K, Schulte F, Tomfohr-Madsen L. Sleep Disturbances in Survivors of Pediatric Acute Lymphoblastic Leukemia and Their Siblings. J Pediatr Psychol. 2020;45(7):707-716.

29. Reynolds CR, Kamphaus RW. Behavior Assessment System for Children (BASC-3). 3rd Edition ed. Bloomington: NCS Pearson Inc.; 2015.

30. Wolfe-Christensen C, Mullins LL, Stinnett TA, Carpentier MY, Fedele DA. Use of the behavioral assessment system for children 2nd Edition: Parent report scale in pediatric cancer populations. Journal of Clinical Psychology in Medical Settings. 2009;16:322-330.

31. Gioia GA, Isquith PK, Guy SC, Kenworthy l. Behavior Rating Inventory of Executive Function (BRIEF2). 2nd Edition ed. Lutz, FL: PAR Inc.; 2015.

32. Kenny DA, Kashy DA. Dyadic data analysis using multilevel modeling. In: Hox JJ, Roberts JK, eds. European Association for Methodology series: Handbook for advanced multilevel analysis. Routledge/Taylor \& Francis Group; 2010:335-370.

33. Hayes AF. Introduction to mediation, moderation, and conditional process analysis. 2nd Edition ed. New York: The Guilford Press; 2017.

34. Choo CC, Chew PKH, Tan P, et al. Health-Related Quality of Life in Pediatric Patients with Leukemia in Singapore: A Cross-Sectional Pilot Study. Int J Environ Res Public Health. 2019;16(12).

35. Hamidah A, Sham Marina M, Tamil AM, et al. Parental reports of behavioural outcome among paediatric leukaemia survivors in Malaysia: a single institution experience. Trop Med Int Health.2014;19(10):1177-1184. 
36. Hile S, Erickson SJ, Agee B, Annett RD. Parental stress predicts functional outcome in pediatric cancer survivors. Psychooncology.2014;23(10):1157-1164.

37. Kunin-Batson A, Kadan-Lottick N, Neglia JP. The contribution of neurocognitive functioning to quality of life after childhood acute lymphoblastic leukemia. Psychooncology. 2014;23(6):692-699.

38. Vannatta K, Gerhardt CA, Wells RJ, Noll RB. Intensity of CNS treatment for pediatric cancer: prediction of social outcomes in survivors. Pediatr Blood Cancer. 2007;49(5):716-722.

39. Kazak AE, Barakat LP, Meeske K, et al. Posttraumatic stress, family functioning, and social support in survivors of childhood leukemia and their mothers and fathers. J Consult Clin Psychol.1997;65(1):120-129.

40. Khalifa AS, Bishry Z, Tantawy AA, et al. Psychiatric morbidity in Egyptian children with acute lymphoblastic leukemia and their care providers. Hematol Oncol Stem Cell Ther. 2014;7(2):76-84.

41. Alderfer MA, Long KA, Lown EA, et al. Psychosocial adjustment of siblings of children with cancer: a systematic review.Psychooncology. 2010;19(8):789-805.

42. Schulte F, Wurz A, Reynolds K, Strother D, Dewey D. Quality of Life in Survivors of Pediatric Cancer and Their Siblings: The Consensus Between Parent-Proxy and Self-Reports. Pediatr Blood Cancer.2016;63(4):677-683.

43. Schulte F, Wurz A, Russell KB, Reynolds K, Strother D, Dewey D. Social adjustment and repressive adaptive style in survivors of pediatric cancer. J Psychosoc Oncol. 2018;36(3):274-286.

Figure Legend FIGURE 1 Theoretical mediation model examining the role of executive function on social adjustment acros

\section{Hosted file}

Table 1_June 182021.docx available at https://authorea.com/users/422155/articles/527910social-adjustment-in-survivors-of-acute-lymphoblastic-leukemia-without-cranialradiation-therapy

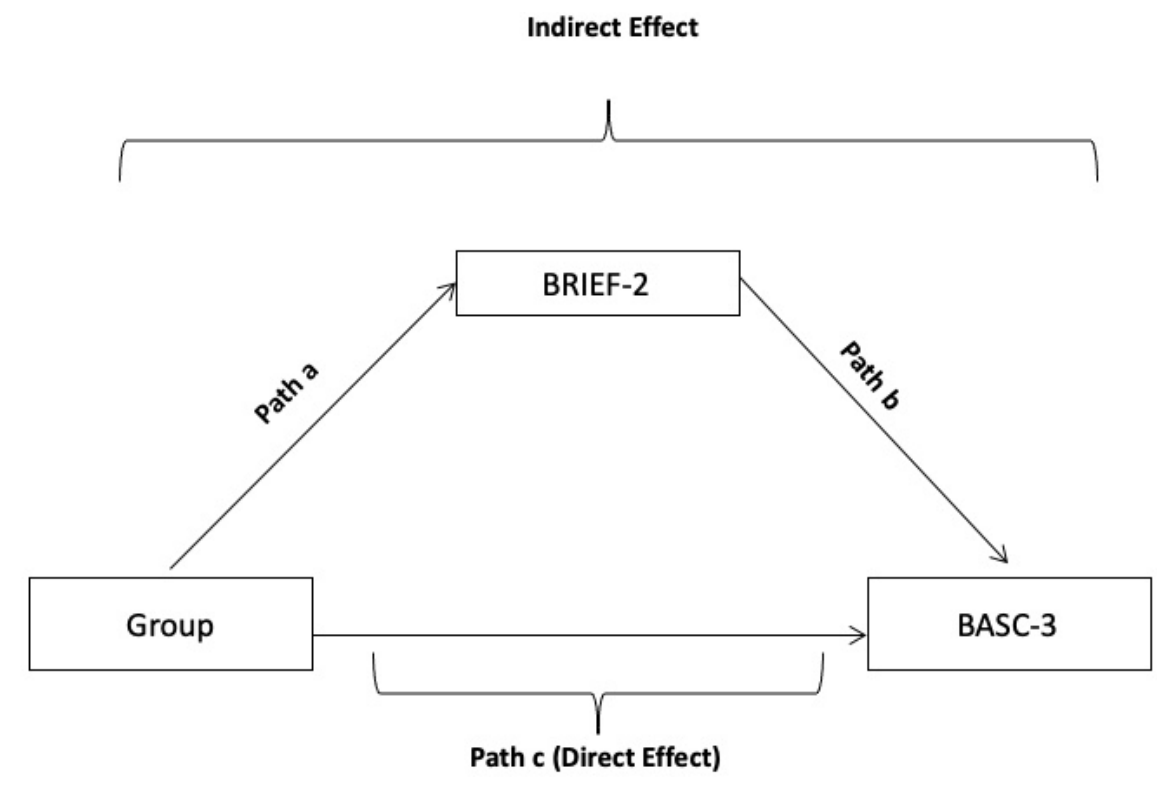

